Revista Paulista de Enfermagem

Como citar este artigo

Moreira ANC, Basile ALO, Aguemi AK.[Capacitação de Diferentes Profissionais na Aplicação da Classificação de Robson] Rev Paul Enferm

[Internet]. 2019;30. doi:10.33159/25959484 repen.2019v30a3

\section{Capacitação de Diferentes Profissionais na Aplicação da Classificação de Robson}

\author{
Teaching Different Health Professionals on the Use of Robson Classification \\ Capacitación de Diferentes Profesionales en la Aplicación de la Clasificación \\ de Robson
}

\section{Alessandra Nascimento Cruz MoreiraI, Anatalia Lopes Oliveira Basile" Adalberto Kiochi Aguemi ${ }^{\mathrm{II}}$}

I Enfermeira obstetra, Centro de Estudos e Pesquisas Dr João Amorim: Endereço: Rua Dr Lund 41 - CEP: 01513-020 - São Paulo, telefone: 34691818 ramal 3321, alessandra.nascimento@ cejam.org.br1,

II Enfermeira obstetra, doutora em Ciências da Saúde, Centro de Estudos e Pesquisas Dr João Amorim: Endereço: Rua Dr Lund, 41 - CEP: 01513-020 - São Paulo, telefone: 34691818 ramal 3324, anatalia.basile@cejam.org.br2,

III Médico obstetra, Secretaria Municipal de Saúde: Endereço: Rua General Jardim, 36 CEP: 01223-010 - São Paulo, telefone: 3397-2226,adalbertoaguemi@prefeitura.sp.gov.br.

\section{RESUMO}

Introdução: A OMS aceitou um sistema proposto por Michael Robson, que agrupa as gestantes em 10 grupos, conforme as características obstétricas. Sendo este sistema de classificação consideravelmente novo, foi necessário capacitar os envolvidos. Método: estudo de intervenção quase experimental por meio de capacitação de assistentes administrativas e supervisão de enfermagem obstétrica. Objetivos: Identificar a facilidade do entendimento dos participantes quanto ao conteúdo teórico da Classificação de Robson apresentada; Quantificar os acertos de casos reais do exercício prático da Classificação de Robson pelos participantes; Identificar a facilidade dos participantes na aplicação prática da Classificação de Robson com o uso do Quadro Direcionador. Resultados: Os resultados mostraram a facilidade de entendimento, quantidade de acertos satisfatórios e facilidade de aplicação da Classificação de Robson após a capacitação. Conclusão: Observamos que o método de capacitação desenvolvida pelos autores possibilitou a realização da Classificação de Robson de forma mais direcionada e simplificada.

Descritores: Classificação; Cesárea; Parto; Informática em Saúde.

\section{ABSTRACT}

Introduction: The $\mathrm{WHO}$ accepted a system proposed by Michael Robson that classifies pregnant women into 10 categories, based on obstetric characteristics. As the system was just recently developed, teaching health professionals involved in caring for women admitted for deliver was required. Method: this 
is a quasi-experimental study that involved training of administrative assistants and obstetric nursing supervision. Aims: to identify participants' ease of understanding of the theoretical content of the Robson classification; to quantify the number of correct answers in practical exercise using the Robson classification; and to identify participants' ease of using the flowchart of the Robson classification in the clinical setting. Results: Health professionals considered the system is easy to understand, answered correctly a satisfactory number of questions, and reported as easy the implementation of the Robson classification after the training. Conclusion: The training method developed by the authors enabled the Robson classification in an objective and simple way.

Keywords: Classification; Cesarean section; Parturition; Medical informatics.

\section{RESUMEN}

Introducción: La OMS aceptó un sistema propuesto por Michael Robson, que agrupa las gestantes en 10 grupos de acuerdo con las características obstétricas. Debido a que este sistema de clasificación es considerablemente nuevo, fue necesario capacitar a los involucrados. Método: estudio de intervención casi experimental llevado a cabo mediante la capacitación de asistentes administrativas y la supervisión de enfermería obstétrica. Objetivos: Identificar la facilidad de comprensión de los participantes con relación al contenido teórico de la clasificación de Robson presentada, cuantificar los aciertos de casos reales del ejercicio práctico de la clasificación de Robson por parte de los participantes, identificar la facilidad de los participantes en la aplicación práctica de la clasificación de Robson mediante el uso del cuadro direccionador. Resultados: Los resultados indicaron la facilidad de comprensión, cantidad de aciertos satisfactorios y la facilidad de aplicación de la clasificación de Robson después de la capacitación. Conclusión: Observamos que el método de capacitación desarrollada por los autores facilitó la realización de la clasificación de Robson de forma mas direccionada y simplificada.

Descriptores: Clasificación; Cesárea; Parto; Informática en salud.

\section{INTRODUÇÃO}

Em 2001 Michael Robson propõe um sistema de classificação que agrupa gestantes em 10 grupos, sistema este aceito pela Organização Mundial de Saúde (OMS), como medida para a redução das taxas de cesáreas ${ }^{1}$.

A Classificação de Robson é um método que se baseia em classificar as gestantes por meio de características obstétricas, como: paridade, parto cesárea anterior, idade gestacional, início do trabalho de parto, apresentação fetal e quantidade de fetos. É uma ferramenta utilizada para monitorar as taxas de cesáreas, sendo uma importante aliada na sua redução. É utilizada de forma relevante e adequada, permitindo a comparação dessas taxas em diferentes hospitais².

Anteriormente para classificar as taxas de cesárea, era considerando apenas a indicação médica para a realização do procedimento, porém essas indicações se tornam divergentes em diversas instituições, por estarem sujeitas a protocolos específicos de cada serviço onde exercem a sua prática ${ }^{3}$.

A prática do uso de uma única classificação de cesárea simplifica a auditoria, o estudo e a comparação das taxas de cesáreas em diferentes aspectos, fornece apoio na criação e implementação de estratégias mais eficazes e voltadas para a redução das taxas de cesáreas ${ }^{4}$, permite monitorar de forma padronizada, para que partindo destes dados coletados, os gestores são incentivados a desenvolver estratégias para reduzi-las ${ }^{5}$, com essa finalidade, torna-se imprescindível capacitar os profissionais para o uso da Classificação dos 10 grupos de Robson.

Os 10 grupos de Robson, é uma base excelente para apoiar as auditorias, é um sistema simples, possível e fácil de ser implantado em qualquer serviço, pois os grupos são bem definidos, são ordenadamente identificados, inclusivos e mutuamente exclusivos ${ }^{6}$, e possibilitam 
acompanhamento e avaliação rotineiras ${ }^{7}$. Para tornar essa afirmativa de forma visual, foi elaborado pelos pesquisadores para este estudo, um Quadro Direcionador para Classificação de Robson (QDCR), com a finalidade de facilitar ao participante aplicar a classificação através de exclusão dos grupos de Robson com menos variáveis. Isso permite que a exclusão das variáveis enquadrem as gestantes no verdadeiro grupo a qual pertence, simplificando a prática da Classificação de Robson, sem fórmulas pré - definidas em tempo real, em lugares onde não há acesso a aplicativos e/ou informática.

O instrumento o QDCR é constituído por 11 linhas e 7 colunas. As colunas foram estabelecidas pelas variáveis obstétricas (paridade, cesárea anterior, tipo de apresentação, início do trabalho de parto, parto múltiplo e Capurro). As linhas foram numeradas segundo os 10 grupos de Robson.

A composição do QDCR, não foi elaborada por ordem numérica crescente ou decrescente, mas sim por grupos de Robson com menos variáveis para classificar, seguindo para os grupos com mais variáveis a considerar.

Os participantes tiveram 40 casos reais de partos, que foram extraídos do livro de parto, para fazer a Classificação de Robson e aplicar os conhecimentos em exercício prático.

Para quantificar, foram contabilizados ao final do exercício prático, os acertos de cada participante.

Segue a baixo o QDCR elaborado para essa capacitação pelas pesquisadoras para classificar as gestantes no grupo a que pertencem no exercício prático.

Quadro 1- Quadro Direcionador para a Classificação de Robson. São Paulo, Brasil- 2016.

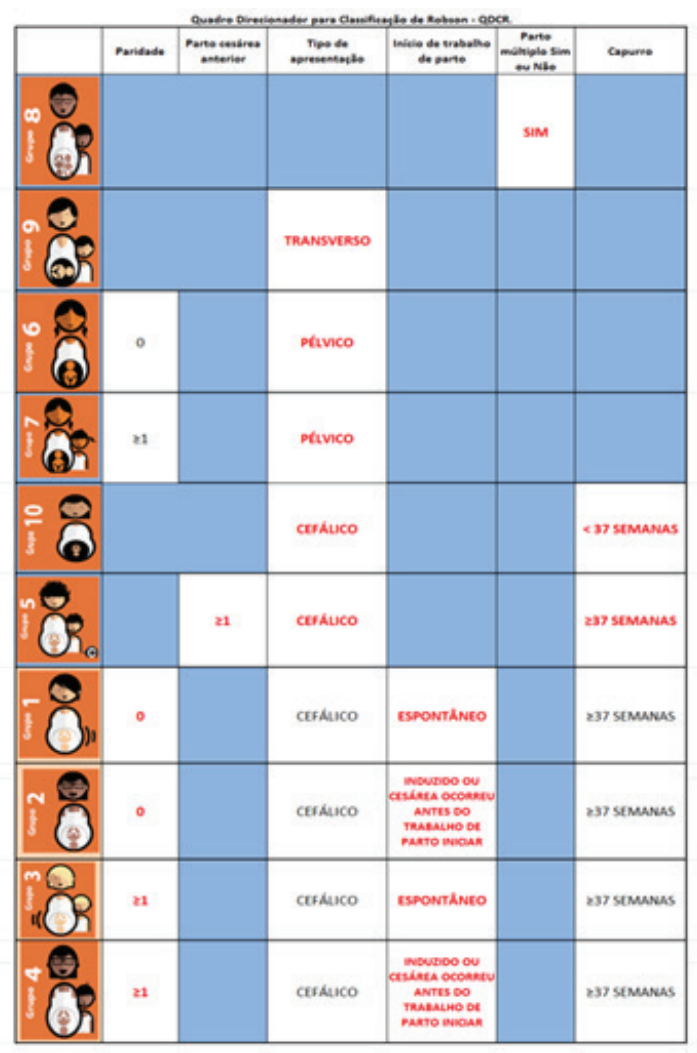

Fonte: Elaborado pelos autores Basile e Moreira. 
Esse estudo é relevante, pois para adotar o método de Classificação de Robson, é fundamental conhecer as particularidades presentes, para isso, torna-se necessário capacitar equipes para que essa classificação seja eficaz.

A justificativa desse estudo é a apresentação de um método de intervenção de capacitação, possibilitando a compreensão dos profissionais para entendimento da Classificação de Robson, possibilitando uma auditoria prática e capaz de direcionar gestores e instituições nas estratégias voltadas nas reduções das taxas de cesáreas.

O presente estudo teve como objetivos: Identificar a facilidade do entendimento dos participantes quanto ao conteúdo teórico da Classificação de Robson apresentada; quantificar os acertos de casos reais do exercício prático da Classificação de Robson pelos participantes e identificar a facilidade dos participantes na aplicação prática da Classificação de Robson com o uso do Quadro Direcionador;

\section{MÉTODO}

Trata-se de estudo de intervenção quase experimental ${ }^{8}$ por meio de capacitação de dois grupos de diferentes profissionais, com abordagem quantitativa.

O estudo foi realizado no Centro de Estudos e Pesquisas Dr. João Amorim (CEJAM), entidade sem fins lucrativos, localizado no município de São Paulo, na região central da cidade de São Paulo. O CEJAM mantém parceria com a Secretaria Municipal de Saúde e Autarquia Hospitalar Municipal, para o gerenciamento do Programa Parto Seguro à Mãe Paulistana, que tem como assistência diferenciada e acolhedora, em oito hospitais da Rede Municipal de Saúde de São Paulo, e tem por objeto do Plano de Trabalho o "atendimento a gestante e ao recém-nascido humanizado, resolutivo, qualificado e humanizado. Que permitem assistência perinatal, o parto e o nascimento seguros sadios e harmoniosos. Além do monitoramento e do acompanhamento sistemático do pós- parto, da mãe e do recém-nato de risco"s.

A população deste estudo foi constituída por colaboradores do CEJAM que exercem funções de assistentes administrativas e de supervisão técnica de enfermagem obstétrica. A amostra foi composta de vinte e três profissionais subdivididos em dois grupos denominados A e B. O grupo A foi formado por assistentes administrativos dos hospitais com Parto Seguro que realizam função de digitação de escalas, tratamento de ponto eletrônico, digitação de indicadores e metas do Plano de Trabalho do Programa Parto Seguro à Mãe Paulistana, não sendo necessário nível superior.

O grupo B foi composto por enfermeiras obstetras, com cargo de supervisoras técnicas, que são profissionais com nível superior e pós-graduação em obstetrícia. Atuam na supervisão técnica e na assistência de enfermagem obstétrica, entre outras funções também condensam os dados mensalmente para o relatório gerencial dos indicadores e metas do Programa Parto Seguro à Mãe Paulistana.

Para essa pesquisa, as variáveis do estudo foram:

A Classificação de Robson - método criado para agrupar gestantes de acordo com as variáveis obstétricas em dez grupos ${ }^{1}$ :

Tabela 1 - Classificação de Robson. 2015.

GRUPO

1 Nulíparas com feto único, cefálico, maior ou igual 37 semanas, em trabalho de parto espontâneo.

2 Nulíparas com feto único, cefálico, maior ou igual 37 semanas, cujo parto é induzido ou que são submetidas à cesárea antes do início do trabalho de parto. 
GRUPO

\section{VARÍAVEIS}

3

Multíparas sem cesárea anterior, com feto único, cefálico, maior ou igual 37 semanas, em trabalho espontâneo.

Multíparas sem cesárea anterior, com feto único, cefálico, maior ou igual 37

4 semanas, cujo parto é induzido ou que são submetidas à cesárea antes do início do trabalho de parto.

5 Todas as multíparas com pelo menos uma cesárea anterior, com feto único, cefálico, maior ou igual 37 semanas.

6 Todas as nulíparas com feto único em apresentação pélvica.

7 Todas as multíparas com feto único em apresentação pélvica, incluindo aquelas com cesárea(s) anterior(es).

8 Todas as mulheres com gestação múltipla, incluindo aquelas com cesárea(s) anterior(es).

9

Todas as gestantes com feto em situação transversa ou oblíqua, incluindo aquelas com cesárea(s) anterior (es).

10 Todas as gestantes com feto único e cefálico, menor que $<37$ semanas, incluindo aquelas com cesárea(s) anterior (es).

Fonte: Organização Mundial da Saúde

A pesquisa ocorreu no período de julho de 2014 a novembro de 2014. Em consonância com os aspectos administrativos e éticos da pesquisa científica, este estudo foi aprovado pelo CEJAM. Essa pesquisa também foi inscrita na Plataforma Brasil conforme Resolução $n^{\circ} 466 / 12$, apreciada e aprovada pelo Comitê de Ética e Pesquisa da Secretaria Municipal de Saúde CAAE n 47950415.4.0000.0086, por tratar-se do parceiro do CEJAM. Os sujeitos participantes desta pesquisa assinaram o termo de consentimento livre e esclarecido antes do início da pesquisa.

Os instrumentos utilizados para pesquisa foram três instrumentos: Facilidade de entendimento dos participantes; facilidade dos participantes na aplicação prática da Classificação de Robson e Quadro Direcionador para Classificação de Robson (QDCR).

Para o desenvolvimento deste estudo foi aplicado conteúdo programático apresentado pelas pesquisadoras em dois módulos: teórico e prático.

O primeiro módulo, o teórico, foi realizada uma apresentação expositiva dialogada em Power Point de onze slides, com duração de 20 minutos. Os slides apresentados introduziram o assunto da classificação desenvolvida por foi Michael Robson, e a definição dos dez grupos, conforme OMS que é contemplado pelas cinco características obstétricas: o início do trabalho de parto, paridade, idade gestacional, apresentação fetal e a quantidade de fetos.

Na sequência, ainda no primeiro módulo foi também desenvolvida atividade com duração de 10 minutos que constou da avaliação dos participantes, quanto ao seu entendimento da parte teórica apresentada, através de preenchimento do instrumento, para mensurar a facilidade de entendimento dos participantes.

A definição da palavra entendimento foi considerada como faculdade pela qual o individuo se apodera das ideias e as compreende ${ }^{10}$.

O segundo módulo ou módulo prático, com duração de 45 minutos, iniciou com explicação breve, mais o exercício prático de classificação de quarenta casos.

Após a realização do exercício prático, os participantes avaliaram a facilidade de aplicação prática da Classificação de Robson, utilizando o QDCR, por meio de preenchimento do 
instrumento, para mensurar a facilidade dos participantes na aplicação prática da Classificação de Robson e o (QDCR). A definição da palavra facilidade foi considerada como qualidade do que é fácil, meio de fazer sem dificuldade ${ }^{10}$.

Os dados foram agrupados em frequência absoluta para realização de cálculos estatísticos e a apresentação na forma de gráfico e tabelas.

\section{RESULTADOS}

A amostra populacional desta pesquisa foi composta por vinte e três colaboradores do CEJAM. O grupo A de cargos administrativos e grupo B enfermeiras obstetras supervisoras, caracterizados em relação à escolaridade e idade conforme tabela abaixo.

Tabela 2 - Descrição da amostra. São Paulo, Brasil- 2016.

\begin{tabular}{|c|c|c|c|}
\hline $\begin{array}{c}\text { Grupos } \\
\text { A e B }\end{array}$ & Idade & Escolaridade & Cargo \\
\hline A & 33 & Ensino médio & Assistente Administrativo Unidade \\
\hline A & 39 & Pós graduação & Encarregado Administrativo \\
\hline A & 37 & Superior Completo & Assistente Administrativo Unidade \\
\hline A & 30 & Superior Completo & Assistente Administrativo Unidade \\
\hline A & 38 & Ensino médio & Assistente Administrativo Núcleo \\
\hline A & 36 & Superior Completo & Assistente Administrativo Unidade \\
\hline A & 33 & Superior Completo & Assistente Administrativo Unidade \\
\hline A & 29 & Ensino médio & Assistente Administrativo Unidade \\
\hline A & 37 & Superior Completo & Assistente Administrativo Unidade \\
\hline A & 33 & Ensino médio & Assistente Administrativo Unidade \\
\hline A & 27 & Superior Completo & Secretária \\
\hline A & 51 & Ensino médio & Assistente Administrativo Unidade \\
\hline $\mathrm{B}$ & 40 & Pós graduação & Supervisora de Enfermagem \\
\hline B & 41 & Pós graduação & Supervisora de Enfermagem \\
\hline B & 47 & Pós graduação & Supervisora de Enfermagem \\
\hline B & 33 & Pós graduação & Supervisora de Enfermagem \\
\hline B & 34 & Pós graduação & Supervisora de Enfermagem \\
\hline B & 37 & Mestre & Supervisora de Enfermagem \\
\hline B & 30 & Pós graduação & Supervisora de Enfermagem \\
\hline B & 44 & Mestre & Supervisora de Enfermagem \\
\hline B & 37 & Pós graduação & Supervisora de Enfermagem \\
\hline B & 32 & Pós graduação & Supervisora de Enfermagem \\
\hline $\mathrm{B}$ & 31 & Pós graduação & Supervisora de Enfermagem \\
\hline
\end{tabular}

Fonte: Dados da pesquisa.

O grupo A constou de 5 (42\%) participantes com escolaridade de nível médio; 6 (50\%), participantes com nível superior e 01 (8\%) participante com pós-graduação, somando um total de doze participantes.

O grupo B constou de 9 (81,81\%) participantes com especialização Lato Sensu e 2 (18,18\%) de mestres, num total de onze participantes. A média de idade dos grupos A e B foram de 35,6 anos.

Segue abaixo tabela 3, com instrumento aplicado, segundo o entendimento dos participantes dos 10 grupos da Classificação de Robson no módulo teórico. 
Tabela 3 - Instrumento de Facilidade de entendimento, com a somatória do entendimento dos participantes, quanto aos 10 grupos da Classificação de Robson. São Paulo, Brasil-2016.

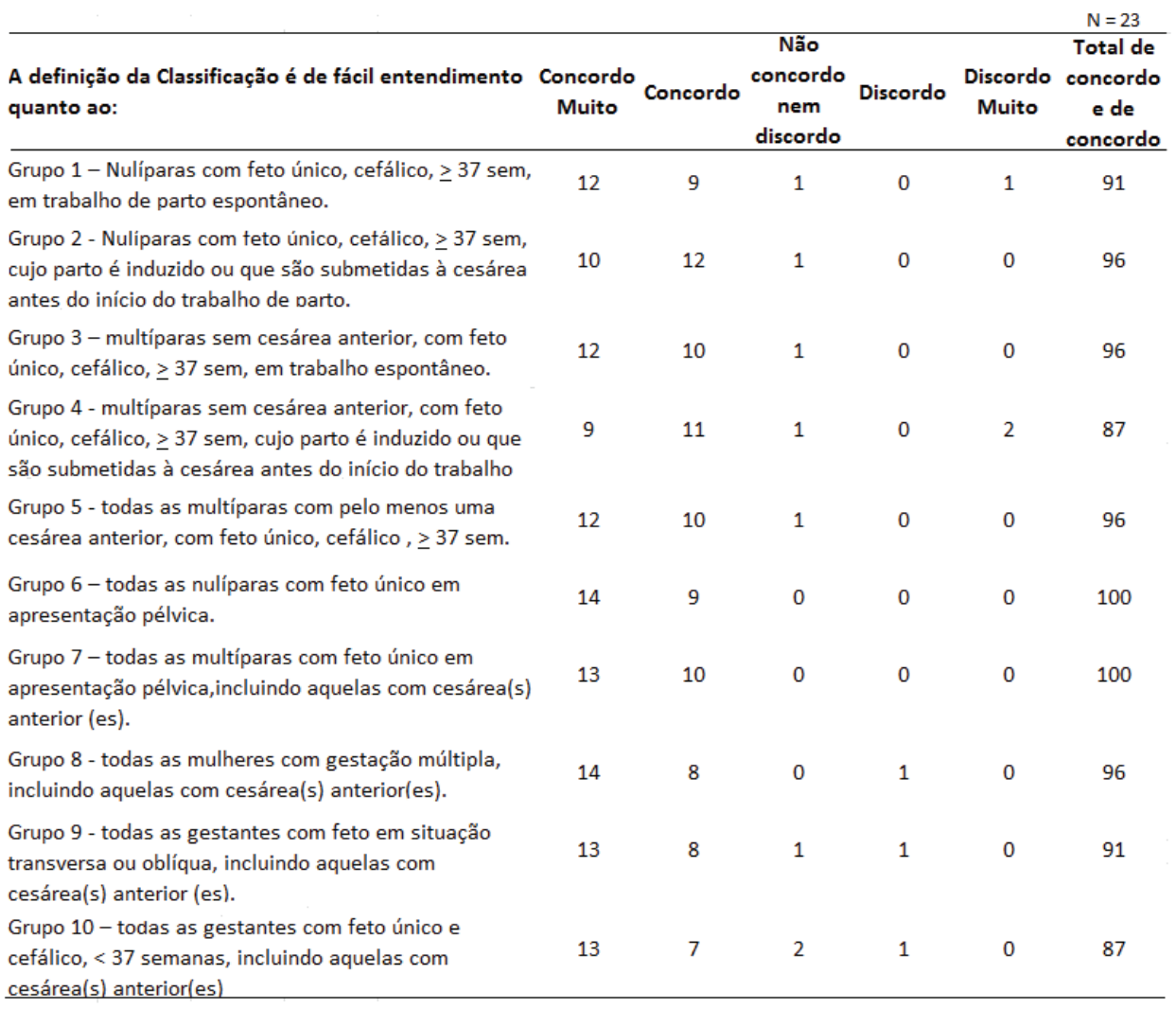

Fonte: Dados da pesquisa.

Para identificar a facilidade do entendimento dos participantes quanto ao conteúdo teórico da Classificação de Robson, o instrumento aplicado demonstrou que $94 \%$ em média dos participantes concordaram com a facilidade de entendimento respondendo o instrumento com afirmações concordo e concordo muito, após apresentação teórica independentemente da quantidade de variáveis dos grupos apresentados.

Ao quantificar os acertos dos participantes em realizar o exercício prático dos 40 casos da Classificação de Robson, percebemos que todos participantes conseguiram classificar os partos utilizando o QDCR, com média de acerto importante, conforme demonstra o Teste T no gráfico 1.

Segundo o Teste T, considerando um nível de confiança de $95 \%$, p-valor 0,01, há evidências estatisticas para apoiar a hipótese de que em média os acertos foram superiores a $90 \%$, independentemente da categoria profissional avaliada.

Para identificar a facilidade de aplicação prática na realização da Classificação de Robson feita pelos participantes, o instrumento demonstrou que alguns grupos são mais fáceis de serem classificados que outros, uma vez que para a realização da Classificação devemos levar em conta as variáveis obstétricas, ou seja, quanto menor for a quantidade de variáveis a considerar, mais fácil é a classificação. 
Gráfico 1. Boxplot-Teste T, distribuição dos acertos dos casos reais do exercício prático da Classificação de Robson realizados pelos participantes. São Paulo, Brasil-2016.

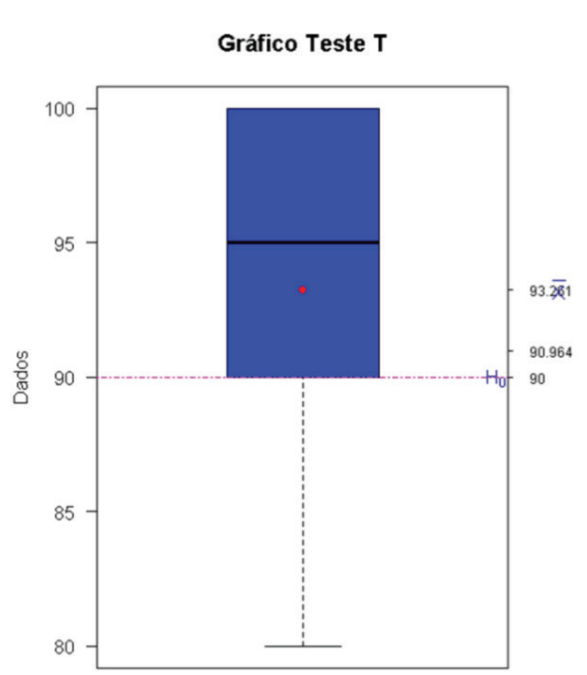

$\mathrm{H}_{0}: \mu \leq 90$

$\mathrm{H}_{1}: \mu>90$

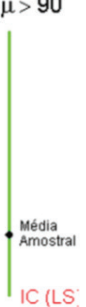

\begin{tabular}{|cc|}
\hline Informações & \\
\hline Estatística T & 2,437699 \\
Graus de Liberdade & 22 \\
P-valor & 0,01 \\
Média Amostral & 93,26087 \\
Desvio Padrão Amostral & 6,415304 \\
Tamanho da Amostra & 23 \\
Hipótese Alternativa Maior que & 90 \\
Nivel de Confiança & $95 \%$ \\
Limite Inferior & 90,96387 \\
Limite Superior & Inf \\
\hline
\end{tabular}

Fonte: Dados da pesquisa

Tabela 4 - Instrumento de Facilidade de aplicação dos participantes na aplicação prática da Classificação de Robson com o uso do Quadro Direcionador, segundo os 10 grupos. São Paulo, Brasil-2016.

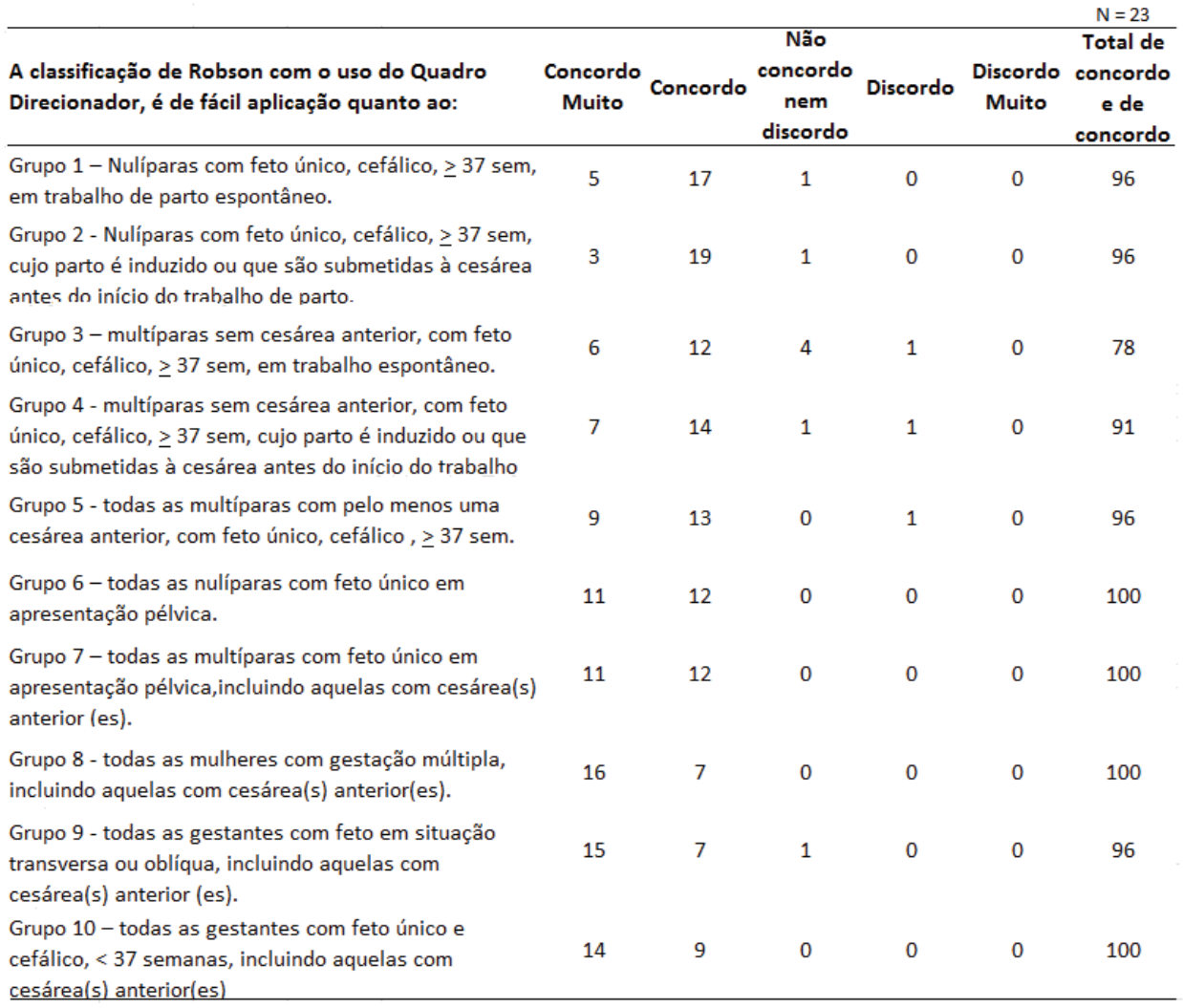

Fonte: Dados da pesquisa. 


\section{DISCUSSÃO}

Em artigos que relatam capacitações de profissionais envolvidos em um processo, foi evidenciado que é extremamente relevante que os envolvidos devam ser capacitados para compreenderem toda a dinâmica e contribuírem para a resolução de algumas situações, pois o desconhecimento e o uso incorreto de suas especificidades são fatores que contribuem para erros ${ }^{11}$.

A descrição da amostra identifica a formação dos grupos por profissionais técnicos e administrativos.

Os resultados obtidos através de instrumento aplicado permitiu conhecer a opinião dos participantes, em relação à afirmação feita pelos autores referente à compreensão e a aplicação da Classificação de Robson. Afirmação essa de que as definições da Classificação dos 10 Grupos de Robson ${ }^{1}$ foram de fácil compreensão e que a aplicação prática também foi simples, com a utilização do QDCR.

Ficou notório que as variáveis apresentadas pelo quadro da OMS são de fácil entendimento, independentemente se a pessoa é técnica ou não na área obstétrica, pois elucida as variáveis através de desenhos específicos e autoexplicativos, possibilitando uma maior compreensão do leitor, em concordância com alguns artigos que relatam que a capacitação para ser assertiva e eficiente, deve ser desenvolvida baseada na população a ser alcançada ${ }^{11}$.

Em concordância com alguns autores que descrevem que o impacto da capacitação provocam nos participantes habilidades adquiridas por receberem conhecimentos, e habilidades úteis para a realização de sua prática ${ }^{12}$, a tabela 3 evidenciou que o uso do QDCR possibilitou, com as variáveis mais relevantes para cada grupo, ficou mais fácil, ágil e simples a aplicação da Classificação de Robson, por conhecer qual variável de destaque, para poder classificar a gestante, afirmação essa considerada por $78 \%$ a $100 \%$ dos participantes, mensurados por meio de instrumento aplicado, por possibilitar simplificar o raciocínio da classificação de forma rápida.

O exercício prático proposto aos participantes, demonstrado no gráfico 1, Bloxplot teste T, apresentou acertos contabilizados dos participantes em 40 casos reais, utilizando o QDCR, e demonstra acertos entre $80 \%$ a $100 \%$ dos participantes. Corroborando com alguns artigos, os participantes deste estudo indicaram que os conteúdos e os instrumentos utilizados tiveram impacto positivo na realização da tarefa proposta ${ }^{13}$, e apenas uma pequena minoria apresentou alguma dificuldade relacionada a conceitos obstétricos, não somente a quantidade de variáveis.

\section{CONCLUSÃO}

A análise da classificação das cesáreas, através dos 10 grupos de Robson, permite conhecer o perfil das gestantes atendidas e conhecer o grupo que mais contribui para as altas taxas de cesárea, para que o gestor possa intervir diretamente com a finalidade na redução dessas.

Sabemos que para que isto ocorra, os profissionais envolvidos nesse processo devem conhecer este novo método para aplicá-lo em diferentes momentos e por diferentes profissionais, para garantir que ações serão tomadas visando à qualidade da assistência para o binômio.

O método de capacitação desenvolvida pelos autores, através de módulo teórico e prático, possibilitou a realização da Classificação de Robson de forma mais direcionada e simplificada, permitindo um melhor entendimento e aplicação. O QDCR facilitou a assimilação dos diferentes profissionais de forma adequada e com grande aproveitamento mesmo para os profissionais que não possuem conhecimento específico em obstetrícia.

O presente estudo tem como principal limitação o fato de ter sido realizado em uma pequena amostra, o que dificulta a universalização dos resultados, entretanto a experiência exitosa na realização deste método de capacitação possibilitou reconhecer que os resultados 
foram satisfatórios para a realização da Classificação dos 10 grupos, podendo ser estimuladas novas pesquisas sobre $o$ assunto.

Conclui-se que o método de capacitação aplicada pelos pesquisadores possibilitou que os profissionais participantes dessa pesquisa compreendessem a Classificação de Robson e conseguissem aplicá-la de forma mais direcionada e simplificada.

\section{REFERÊNCIAS}

1. Organização Mundial da Saúde. Organização Pan-Americana da Saúde. Declaração da OMS sobre taxas de cesáreas. Disponível em: www.paho.org/bra.

2. Betran AP, Gulmezoglu $M$, Robson $M$, Merialdi $M$, Souza JP,Wojdyla D, Widmer $M$, Carroli G, Torloni MR, Langer A, Navaez A,Velasco A, Faúndes A, Acosta A,Valladares E, Romero M, Zavaleta N, Reynoso S, Bataglia V.WHO Global Survey on Maternal and Perinatal Health in Latin America: classifying caesarean sections. BioMed Central. Reproductive Health. 2009 out; 6:18. Disponível em http://www.biomedcentral.com/ content/pdf/1742-4755-6-18.pdf.

3. Dias MAB, Deslandes SF. Cesarianas: percepção de risco e sua indicação pelo obstetra em uma maternidade pública no Município do Rio de Janeiro. Cad. Saúde Pública, Rio de Janeiro, jan-fev, 2004; 20(1):109-116.

4. Torloni MR, Betran AP, Souza JP, Merialdi M, Widmer M, Allen T, Gulmezoglu M. Classifications for cesarean section: A systematic Review. Plos One. 2011 jan; 20;6(1). Disponível em: http://www.ncbi.nlm.nih.gov/pubmed/21283801.

5. Guerreiro C. A propósito do Artigo "Indicações para cesarianas num hospital terciário durante 7 anos". Acta Med Port 2013 Nov-Dec ;26(6):630-632. Disponível em: http://repositorio.chlc.min-saude.pt/bitstream.

6. Veríssimo CA, Gomes RF, Matozinhos M, Matos T,Sepúlveda F, et al. Implementação dos 10 group Classification System: compreender o parto cesariana. Acta Obstet Ginecol Port 2013; 7 (1):3-7. Disponível em: http: //www.fspog.com/fotos/ editor2/2013 1estudo_original.pdf.

7. Vogel JP, Betrán AP, Vindevoghel N, Souza JP, Torloni M R, Zhang J,et al. Use of the Robson classification to assess caesarean section trends in 21 countries: a secondary analysis of two WHO multicountry surveys. Lancet Glob Health 2015 abr; 3: e260-70. Disponível em: www.ncbi.nlm.nih.gov/pubmed/25866355.

8. Sampieri RH, Collado CF, Lucio MDPB. Metodologia de Pesquisa. $5^{\text {a }}$ Edição. Porto Alegre: Penso;2013. p.167.

9. Centro de estudos e pesquisas Dr. João Amorim. Sobre o CEJAM. [Acesso em: 10 jun. 2015].Disponível em: http://cejam.org.br/index.php?pg=isnt_sobre\&fnc=visual.

10. Ferreira $\mathrm{ABH}$. Dicionário Aurélio Básico da Língua Portuguesa. Rio de Janeiro: Nova Fronteira. Disponível em www.dicionarioaurelio.com.

11. Melo DO, Molino CGR, Ribeiro E, Lieber NSR. Capacitação e intervenções de técnicos de farmácia na dispensação de medicamentos em Atenção Primária à Saúde. Rev Associação Brasileira de Ciência e Saúde Coletiva. 2015 ago 22(1).Disponível em: http://www.scielo.br/pdf/csc/v22n1/1413-8123-csc-22-01-0261.pdf.

12. Barboza VV, Klijn TP, Paravic T, Molina AS, Carrillo KLS. Eficácia de uma intervenção personalizada de aconselhamento em enfermagem, presencial e telefônica, para fatores de risco cardiovascular: ensaio clínico controlado. Rev. Latino-Am. Enferm. 2016 jan; 24(2747). Disponível em: www.revistas.usp.br/rlae.

13. Freitas CPP, HabigzangII LF .Percepções de psicólogos sobre a capacitação para intervenção com vítimas de violência sexual. Psicologia Clínica, Jan-Jun 2013; 25(2). Disponível em: pepsic.bvsalud.org/scielo. 